\title{
Downstream relaxation of velocity profiles in pipe-flow with swirl disturbances
}

\author{
Stromabwärts-Relaxation von Geschwindigkeitsprofilen mit Drallstörungen
}

DOI 10.1515/teme-2016-0029

Received August 11, 2016; revised October 7, 2016; accepted

October 12, 2016

\begin{abstract}
We study characteristic flow patterns downstream of a standardized swirl disturbance generator using laser-Doppler velocimetry (LDV). To investigate the spatial development of flow patterns, we conduct LDV measurements in cross-sections located at various distances downstream from the swirl disturbance generator. Focusing on velocity profiles, decay of swirl, and performance indicators used to describe the characteristic shape of the velocity profiles, we systematically compare the experimental results with available references and various theories for decay of swirl disturbances. We find that the standardized swirl disturbance generator provides exponentially decaying swirling flow that is best captured by the theory of Steenbergen and Voskamp ${ }^{1}$. In addition, deviations from the axial reference profile caused by the swirl disturber persist for long downstream distances. In particular, our results suggest that the peakness factor relaxes linearly towards the fully-developed state.
\end{abstract}

Keywords: Laser-Doppler velocimetry, swirl disturbance generator, swirling pipe-flow, performance indicators, downstream relaxation.

1 Steenbergen W, Voskamp J (1998) The rate of decay of swirl in turbulent pipe flow. Flow Meas Instr 9:67-78

\footnotetext{
*Corresponding author: Denis F. Hinz, Kamstrup A/S, Industrivej 28 Stilling, 8660 Skanderborg, Denmark, e-mail: dfh@kamstrup.com

Simon Graner: Kamstrup A/S, Industrivej 28 Stilling, 8660 Skanderborg, Denmark

Christian Breitsamter: Lehrstuhl für Aerodynamik und Strömungsmechanik, Technische Universität München, Boltzmannstrasse 15, 85748 Garching b. München, Germany
}

Zusammenfassung: Wir untersuchen charakteristische Strömungsmuster hinter einem standardisierten Drallgenerator mittels Laser-Doppler-Anemometrie (LDA). Um die räumliche Entwicklung von Strömungsmustern $\mathrm{zu}$ untersuchen, führen wir LDA-Messungen in Querschnitten in unterschiedlichen Abständen stromabwärts des Drallgenerators durch. Mit Fokus auf Geschwindigkeitsprofilen, dem Verfall von Drallstörungen, und Performance-Indikatoren, um die charakteristische Form der Geschwindigkeitsprofile zu beschreiben, vergleichen wir systematisch die experimentellen Ergebnisse mit verfügbaren Referenzen und verschiedenen Theorien für den Zerfall von Wirbelstörungen. Unsere Ergebnisse zeigen, dass der standardisierte Drallgenerator exponentiell abklingende Drallströmungen erzeugt, die am besten durch die Theorie von Steenbergen and Voskamp ${ }^{1}$ erfasst wird. Außerdem können die durch den Störkörper verursachten Abweichungen vom axialen Referenzprofil lange stromabwärts nachgewiesen werden. Insbesondere legen unsere Ergebnisse nahe, dass der sogenannte Peakness-Faktor linear zu einer voll entwickelten Strömung relaxiert.

Schlüsselwörter: Laser-Doppler-Anemometrie, Drallstörungen, Drallgenerator, wirbelnde Rohrströmung, Performance-Indikatoren, Stromabwärts-Relaxation.

\section{Introduction}

Swirling flow occurs in many technical configurations of practical relevance and can be either purposefully created, for example, to increase mixing in combustion chambers, or it may be an undesirable and spurious effect, for example, in flow measurement applications where an undisturbed nearly fully developed flow profile can be advantageous to achieve good accuracy. In most pipe-flow applications, swirling flow patterns are generated by various installation elements including (double) bent pipes and valves. These spurious swirling flows may have severe 
impacts on the accuracy of instrumentation devices like flow meters, heat meters, and other sensors.

In general, reliable and accurate estimates for the spatial and temporal development of swirling flow patterns are relevant for many engineering applications including combustion, chemical and fusion reactors, turbomachinery, control devices, and drinking water supply as well as hot water supply in district heating networks. The downstream development of flow patterns is the essential feature for most applications involving swirling flow. Knowing how far downstream swirling flow persists is critical for applications with purposefully created, and spurious swirling flows alike. In addition to the spatial development, changes in the shape of axial velocity profiles induced by swirl are pertinent in particular for flowmetering applications, where the accuracy of meters may depend on the axial velocity profile. This influence is prescribed through the inherent coupling of axial and radial flow components in the momentum balance of the NavierStokes (NS) equations.

Early investigations of swirling pipe-flow date back to the 50's and 60's. Talbot [33] provides a theoretical analysis of the laminar case with a weakly swirling velocity profile. Reverse axial flow in the centre of the pipe in a swirling flow is first observed by Nutall [22]. The experiments of Binnie [3] verify that swirl can give rise to reversed axial flow in the core region of pipe-flow, which reflects an extreme case of the inherent coupling of axial and radial flow to satisfy the momentum balance. Further experiments are performed by Sachdeva [28], who obtained a reverse axial flow in an annular region using tangential inlets to generate the swirl. A set of experiments by Wolf Jr. et al. [36] shows a flat axial velocity profile and an almost linear tangential velocity profile except in the region close to the wall. An experimental description on similarity properties and the effect of swirl on turbulence, flow development, and effects of flow reversal is provided by Yajnik and Subbaiah [37].

Koch [16], Kreith and Margolis [17], and Smithberg and Landis [30] investigate effects of swirl on skin friction and heat transfer, finding that the surface heat transfer coefficients in swirling flow are at least four times as large as in purely axial flow and highly dependent on the swirling intensity, the fluid density, and the temperature gradient. Similarly, Migay and Golubev [19] investigate the possibility of increasing the heat transfer rate by adding swirl and Algifri et al. [2] test empirical approximations to predict associated heat transfer coefficients. Along with heat transfer effects and flow reversal, various studies encompassed the decay of swirl disturbances and the associated universalities between different types of swirl.
In this article, we study flow patterns generated with a standardized swirl disturbance generator (EN ISO 40642:2014 [7] and OIML R 49-2:2013 [23], Figure 2 (b)) that is commonly used for type approval tests in the flow measurement industry. To investigate the spatial development and the decay of the swirling wake, we collect LDV data of all three velocity components at various measurement sections downstream from the swirl disturbance generator. Moreover, we assess the potential of analytical theories of Steenbergen and Voskamp [32], Gersten and Papenfuss [11], and Murakami et al. [21] to approximate our experimental results. Besides the decay of the swirling intensity, we also study the downstream development of the axial flow profile using performance indicators and compare the results to experimental particle-image velocimetry (PIV) studies of Eichler and Lederer [6] with a DN80 pipe and to LDV results of Müller et al. [20] for a DN20 pipe. Before continuing the discussion of swirl decay, we first review different dimensionless measures of the swirling intensity.

\subsection{Dimensionless measures of the swirling intensity}

Within investigations of swirl decay, various integral metrics to measure and describe the swirling intensity have been proposed. We summarize the available dimensionless swirl numbers in Table 1 (notice that Equations (1) to (7) are included in Table 1). The velocity components used in the various definitions are illustrated in Figure 1. The dimensionless swirl number $S$ (Equation (1) in Table 1) is the ratio of the azimuthal momentum flux to the axial momentum flux, where $\bar{w}$ and $\bar{v}_{\theta}$ are the axial and tangential velocity components, $R$ is the pipe radius, and an overbar denotes averages over repeated single-point measurements (that is, LDV bursts), as discussed in Section 2. Through several assumptions an alternative swirl number $S^{*}$ (Equation (2) in Table 1) is provided, where

$$
w_{\mathrm{vol}}=\frac{4 Q}{\pi D^{2}}
$$

is the volumetric velocity. Notice that Equations (1) and (2) in Table 1 are formulated for integration over one measurement path. For grid measurements as in the present study, the swirl number and other performance indicators can be averaged over the different measurement paths as discussed in Section 2.2. Different Rossby numbers Ro and Ro* (Equations (3) and (4) in Table 1) are used by Yajnik and Subbaiah [37] and Steenbergen and Voskamp [32]. Further, a dimensionless swirl intensity $\Gamma$ (Equation (5) in Table 1) is defined by Ito et al. [13], where $U_{\theta w}$ is the 
Table 1: Different measures for swirling intensity.

\begin{tabular}{|c|c|c|}
\hline Swirl number definition & & References \\
\hline$S=\frac{\int_{0}^{R} r^{2} \bar{w} \bar{v}_{\theta} \mathrm{dr}}{R \int_{0}^{R} \bar{w}^{2} r \mathrm{dr}}$ & (1) & $\begin{array}{l}\text { Senoo and Nagata [29], Yajnik and Subbaiah [37], Khalatov [14], Algifri [1], } \\
\text { Parchen [24], Rocklage-Marliani et al. [27] }\end{array}$ \\
\hline$S^{*}=\frac{\int_{0}^{R} r^{2} \bar{w} \bar{v}_{\theta} \mathrm{dr}}{R^{3} w_{\mathrm{vol}}^{2}}$ & (2) & $\begin{array}{l}\text { Murakami et al. [21], Kitoh [15], Yu and Kitoh [40], Steenbergen and Parchen [31], } \\
\text { Steenbergen and Voskamp [32], Gersten and Papenfuss [11], Rocklage-Marliani et al. [27] }\end{array}$ \\
\hline Ro $=\frac{\omega D}{2 w_{\text {vol }}}$ & (3) & Yajnik and Subbaiah [37] \\
\hline $\mathrm{Ro}^{*}=\frac{\omega D}{4 w_{\mathrm{vol}}}$ & (4) & Steenbergen and Voskamp [32] \\
\hline$\Gamma=\frac{U_{\theta w}}{R \omega_{i}}$ & (5) & Ito et al. [13] \\
\hline$N=\frac{\bar{v}_{\max }}{w_{\mathrm{vol}}}$ & (6) & Genc et al. [9] \\
\hline$\phi=\operatorname{atan} \frac{\left|\bar{v}_{x y}\right|_{\max }}{w_{\mathrm{vol}}}$ & (7) & [38] \\
\hline
\end{tabular}
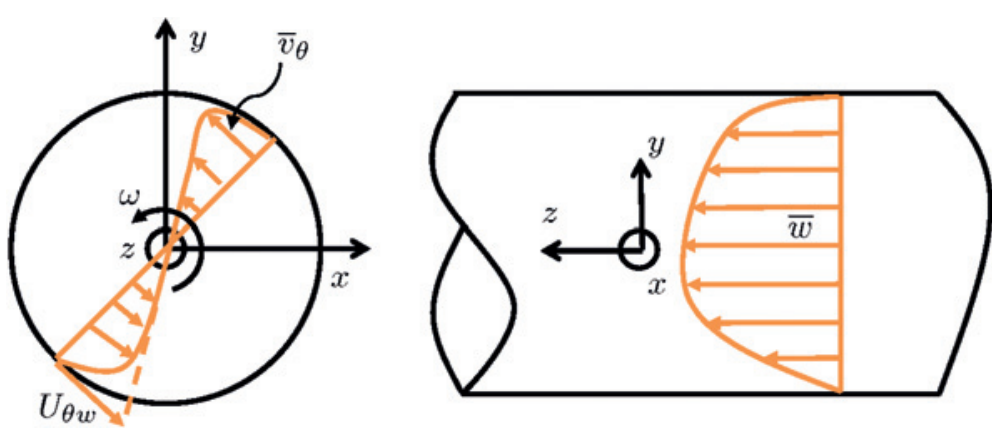

Figure 1: Illustration of different velocities used in the various measures for swirling intensity summarized in Table 1. extrapolated value of the tangential velocity at the pipe wall, and $\omega_{i}$ is a reference angular velocity at the inlet. Notice that (5) is formally similar to the inverse of (4). The swirl number $N$ (Equation (6) in Table 1) is used by Genc et al. [9], where $\bar{v}_{\max }$ is the maximal velocity of the secondary flow field. This swirl number is similar to the definition of the swirl angle (10), where

$$
\bar{v}_{x y}=\sqrt{\bar{u}^{2}+\bar{v}^{2}}
$$

is the magnitude of the velocity component in the $x y$ plane. Also see Section 3.2 for the definition of the swirl angle and additional performance indicators. The swirl angle $\phi$ (Equation (7) in Table 1) is commonly used in standards related to flow measurement (see, for example,
EN ISO 5167-1:2003 [8]) and Steenbergen and Voskamp [32] provide a review of the similarities and differences between the swirl angle (7) and the swirl number (2).

In view of the established standards in the flow measurement industry, we focus on the swirl angle

$$
\phi=\operatorname{atan} \frac{\left|\bar{v}_{x y}\right|_{\max }}{w_{\mathrm{vol}}},
$$

where $\left|\bar{v}_{x y}\right|_{\max }$ is the maximum of the magnitude of the secondary velocity. This also facilitates a meaningful comparison with the recent experiments of Eichler and Lederer [6] and Müller et al. [20]. 


\subsection{Decay of swirl}

Based on measures of the swirling intensity discussed in Section 1.1, various experimental observations and theoretical analyses suggest that swirl in a straight pipe decays exponentially.

Most theories aimed at swirl decay are contingent on estimations of the wall shear stress or simplifications of the NS equations. Formed on early experimental observations, Kreith and Sonju [18] develop a theory that yields an exponential decay for turbulent swirling flow, granted the assumption of an axial mean velocity profile with a small perturbation and an eddy-viscosity for the tangential direction. Senoo and Nagata [29] derive an exponential law for the decay of $S$ through differentiating (1) with respect to the pipe length and making additional assumptions on the decay of the axial momentum flux. Similarly, Ito et al. [13] determine the decay associated with (5) as

$$
\Gamma_{\omega}^{*}=\gamma e^{-\kappa\left(\frac{z}{R}\right)\left(\frac{v}{R^{2} \omega}\right)},
$$

where $v$ is the kinematic viscosity, $\kappa$ is the dimensionless decay rate that is determined from experimental results, $z$ is the axial coordinate, and $\gamma$ is a dimensionless parameter that depends on the inlet boundary conditions.

While the theories of Senoo and Nagata [29] and Ito et al. [13] rely on parameters that are estimated empirically from specific experimental results, other theories focus on establishing general relationships for estimating the associated decay coefficient theoretically or semi-empirically. Distinguishing various effects of boundary conditions, the theory of Murakami et al. [21] simplifies the incompressible NS equations for a steady and axisymmetric flow, providing an ordinary differential equation (ODE) for the rate of change of the swirl number (2)

$$
\mathrm{d} S^{*} / \mathrm{d}(z / D)=-2 k \lambda V_{\Theta_{0}} V_{0},
$$

where $k$ is a dimensionless constant and $V_{\Theta_{0}}=\bar{v}_{\theta} / w_{\text {vol }}$ and $V_{0}=\left(V_{z_{0}}^{2}+V_{\Theta_{0}}^{2}\right)^{1 / 2}$ are the velocity ratios outside the wall layer with $V_{z_{0}}=\bar{w} / w_{\mathrm{vol}}$. With the assumption that the swirl intensity is proportional to the product $V_{\Theta_{0}} V_{0}$, the ODE (12) can be solved analytically, yielding the exponential decay law

$$
S^{*}=S_{0}^{*} e^{-\beta(z / D)},
$$

where the exponent $\beta$ is the decay rate and $S_{0}^{*}$ the initial swirl intensity at $z=0$. Comparison of (12) and (13) yields an explicit expression for the decay rate

$$
\beta=2 k \lambda V_{\Theta_{0}} V_{0} / S^{*}
$$

as a function of the roughness $\lambda$ of the pipe wall and flow conditions that can be estimated from experiments.

Subsequent investigations establish systematic estimates for the dependency of the decay rate $\beta$ on the friction factor $\lambda$ and the Reynolds number Re. For example, calculations of Reader-Harris [26] suggest that $\beta$ is directly proportional and close to identical to $\lambda$. Recognizing different vortex types appearing in swirling flow (solid body, concentrated vortex, and wall jet), Steenbergen and Voskamp [32] identify a decrease of $\beta$ with increasing Reynolds numbers and a similarity of the change of $\beta$ and $\lambda$ with respect to Re. Through theoretical analysis based on simplifications of the incompressible NS equations, Gersten and Papenfuss [11] find that $\beta=\lambda$ for the special case of a single longitudinal vortex.

\section{Materials and methods}

We perform all experiments on a verification and calibration test bench in the flow laboratory of Kamstrup A/S using brass pipes of inner diameter $D=15.0 \mathrm{~mm}$ and a water temperature of $T=20.0^{\circ} \mathrm{C}$ corresponding to a kinematic viscosity $v=1.004 \cdot 10^{-6} \mathrm{~m}^{2} / \mathrm{s}$. The volumetric flow rate $Q$, the water temperature $T$, and the pressure $p$ are stabilized within a PID feedback loop. We verify the stability of $Q, T$, and $p$ by logging data from the master meters and the corresponding temperature and pressure sensors. Let $\bar{Q}_{M}$ denote the time-averaged master flow-rate and let $\sigma_{Q_{M}}$ denote the associated standard deviation of $\bar{Q}_{M}$ in time providing a measure for the stability of the flow-rate. We find $\sigma_{Q_{M}} / \bar{Q}_{M} \approx 0.25 \%$ and that the master meter signal has the characteristics of random white noise, which confirms that there is no preferred timescale and no low frequency disturbances that might bias the long-time accuracy of LDV measurements.

We consider Reynolds numbers $\mathrm{Re}=6.0 \cdot 10^{4}, \mathrm{Re}=$ $4.0 \cdot 10^{4}$, and $\operatorname{Re}=1.0 \cdot 10^{3}$, which correspond to volumetric flow rates of $Q=2.556 \mathrm{~m}^{3} / \mathrm{h}, Q=1.76 \mathrm{~m}^{3} / \mathrm{h}$, and $Q=0.0426 \mathrm{~m}^{3} / \mathrm{h}$, where

$$
\operatorname{Re}=\frac{w_{\mathrm{vol}} D}{v}
$$

is the Reynolds number based on the volumetric velocity (8). Further, we conduct LDV measurements in crosssections $10.0 \mathrm{D}, 12.0 \mathrm{D}, 50.0 \mathrm{D}$, and $105.0 \mathrm{D}$ downstream of the swirl disturbance generator, as illustrated in Figure 2 (d)-(f). Flow perturbations are generated at $z=0$ (Figure 2 (d)) with a standardized clockwise swirl disturbance generator according to EN ISO 4064-2:2014 [7] and OIML R 49-2:2013 [23], as shown in Figure 2 (b). The 


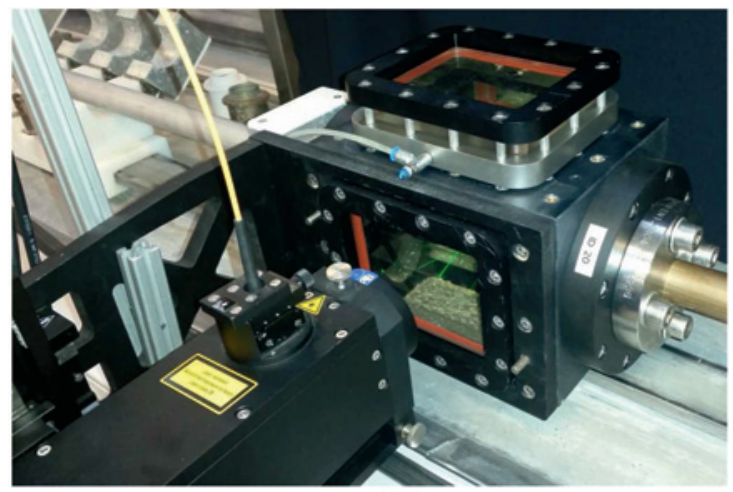

(a)

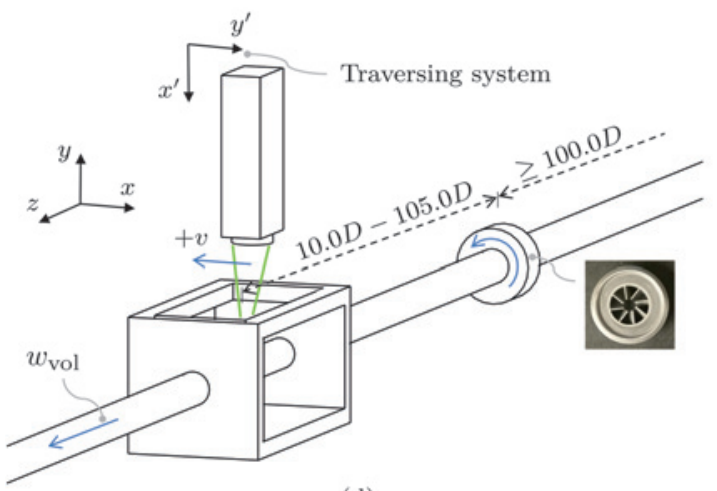

(d)

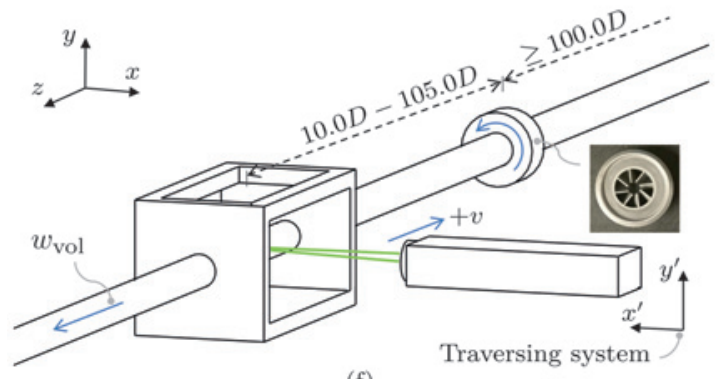

(f)
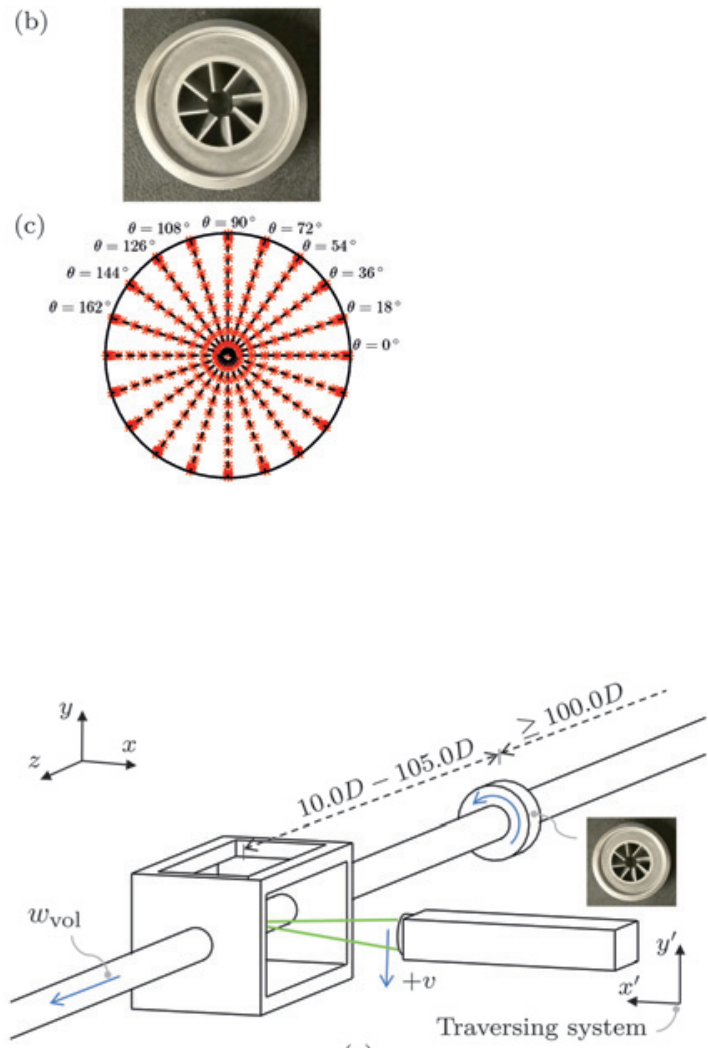

(e)

Figure 2: Clockwise swirl disturbance generator according to EN ISO 4064-2:2014 [7] and OIML R 49-2:2013 [23] (a), the LDV probe (b), the associated LDV measurement grid with coordinate system (c), and illustrations of the experimental setup to measure the $x$ velocity component (d), the $y$ velocity component (e) and the $z$ velocity component (f).

standardized swirl disturbance generator is installed at least $100.0 \mathrm{D}$ downstream from the inlet of the test section to ensure undisturbed fully-developed upstream flow conditions (Figure 2 (d)-(f)).

We use a commercial shifted one-dimensional Nd:YAG LDV probe from ILA/Optolution with a window chamber that enables full three-dimensional optical access for the measurement of all three velocity components (Figure 2(a)). The probe is mounted on a traversing system for automated displacement in a Cartesian coordinate system with origin at the center of the pipe, as illustrated in Figure 2 (d)-(f). The LDV probe measures one velocity component at a time such that the traversing system is installed on the side of the window chamber to measure the axial velocity component as well as the velocity component in $y$-direction and on top to measure the velocity component in $x$-direction. The different measurement positions are illustrated along with the coordinate system in Figure 2 (d)-(f). The flow is seeded with neutrally-buoyant silver-coated hollow glass beads.

The amount of data acquired at each measurement point is determined by the choice of two experimental con- 
straints: (I) the maximal number of single-point samples (that is, LDV bursts) $n_{\max }$ and (II) a timeout $t_{\max }$ for each single-point measurement on the measurement grid. For the present measurements we choose

$$
n_{\max }=10^{3} \text { and } \quad t_{\max }=60.0 \mathrm{~s} .
$$

Axial velocity profiles are obtained through a collection of single-point measurements of the local axial mean velocity $\bar{w}$ over the measurement grid (Figure 2 (c)). To determine $\bar{w}$, we use the estimator

$$
\bar{w}=\frac{1}{n} \sum_{i=1}^{n} w_{i}
$$

with $w_{i}$ single-point samples of velocities and $n$ the number of samples. The associated dimensionless turbulence intensity is

$$
\mathrm{Tu}=\frac{\sigma_{w}}{\bar{w}},
$$

where

$$
\sigma_{w}=\left(\frac{1}{n-1} \sum_{i=1}^{n}\left(w_{i}-\bar{w}\right)^{2}\right)^{1 / 2}
$$

is the standard deviation of samples $w_{i}$. To estimate the reliability of the estimator (17) at each spatial measurement point, we determine the associated standard error

$$
\sigma_{\bar{w}}=\sigma_{w} / \sqrt{n} .
$$

The mean velocity components $\bar{u}$ in $x$ direction and $\bar{v}$ in $y$ direction are determined analogously in two consecutive LDV measurements.

While a detailed discussion of measurement uncertainties goes beyond the scope of the present article, it is important to note that the main contributions to the uncertainty of the velocity determined at a single-point include the uncertainty of the calibrated fringe distance, uncertainties due to turbulence and a finite amount of samples (that is, bursts, see also the discussion of the standard error above), and various geometrical uncertainties. The geometrical uncertainties include uncertainties in the calculations for the ray-tracing and uncertainties in the positioning of the LDV probe and the associated measurement volume at the intersection of the laser beams. By using a traversing system operating in Cartesian coordinates, we obtain positioning uncertainties $\pm \Delta x$ and $\pm \Delta y$ that are constant in the entire measurement cross section. However, an error propagation calculation shows that the positioning uncertainties $\pm \Delta r$ and $\pm \Delta \phi$ in a polar coordinate system depend on the location on the measurement grid. In contrast, a traversing system operating in polar coordinates would provide uncertainties $\pm \Delta r$ and $\pm \Delta \phi$ independent of the location.

\subsection{Reconstruction of velocity profiles}

Optical disturbances and reflections may cause measurement errors leading to corrupted and unreliable data at different measurements points, especially at locations close to the pipe wall. Following the reconstruction procedure of Hinz [12], we identify these invalid data points with a criterion based on the associated standard error (20) of the local axial mean velocity (17). We define $\xi$ as admissible threshold value for standard errors. Points with small enough standard error

$$
\frac{\sigma_{\bar{w}}}{\bar{w}}<\xi
$$

are not reconstructed and the raw data is used. Points that do not fulfill the criterion (21) are reconstructed through interpolation from neighboring points. For the post-processing of all data in this article we choose $\xi=10.0 \%$. If the reconstruction point or several reconstruction points are located next to the wall, the points are reconstructed with a power-law ansatz according to VDI/VDE 2640 Blatt 1 [34] and VDI/VDE 2640 Blatt 2 [35]. To avoid artificial smoothing of our results, we choose a rather large value for the admissible standard error. Consequently, some 'outliers' with large statistical errors may still be contained in the data, in particular for measurements of secondary components with very low velocities (or zero velocities). To keep our results objective, we do not manually correct any such 'outliers'.

\subsection{Computation of performance indicators}

To compare the downstream development of flow profiles quantitatively, we use performance indicators (see, for example, Eichler and Lederer [6]). Performance indicators are useful integral metrics to quantify the shape of flow profiles. Following Yeh and Mattingly [38] and Müller et al. [20], we compute the swirl angle $\phi$, the profile factor $K_{p}$, the asymmetry factor $K_{a}$, and the turbulence factor $K_{\mathrm{Tu}}$. The definition of the performance indicators is provided in the corresponding Sections 3.2.1, 3.2.2, 3.2.3, and 3.2.4. We compute performance indicators for individual profiles, which are defined by the angle $\theta$ in the measurement grid (Figure 2 (c)). Hereupon, we compute the average

$$
K=\frac{\sum_{i=1}^{M} K_{i}}{M},
$$

where $K_{i}$ is the performance indicator of profile $i$ and $M=$ 10 is the amount of individual profiles on the measure- 
ment grid. Further, we determine the associated standard deviation of performance indicators determined from individual profiles on the measurement grid (Figure 2 (c)) analogously to the definition (19) of the standard deviation. In all applicable figures, the standard deviations are shown as error bars. To assess the relaxation towards a fully-developed profile, we include the established limits for a sufficiently fully-developed flow profile according to Eichler and Lederer [6]:

$$
\begin{array}{ll}
\text { Swirl angle } \phi: & \phi_{\max }=2^{\circ} \\
\text { Profile factor } K_{p}: & 0.8 \leq K_{p} \leq 1.3 \\
\text { Asymmetry factor } K_{a}: & K_{a, \max }=1.0 \% \\
\text { Turbulence factor } K_{\mathrm{Tu}}: & K_{\mathrm{Tu} \text {,max }}=2.0
\end{array}
$$

\section{Results}

\subsection{Velocity profiles}

Contour plots of the axial velocity component $\bar{w}$ and the magnitude (9) of the secondary flow are shown in Figure 3 for various measurement cross-sections and Reynolds numbers. In the measurement sections at $10.0 \mathrm{D}$ and $12.0 \mathrm{D}\left(\mathrm{Re}=4.0 \cdot 10^{4}\right)$, we find an axial velocity profile with a nearly flat core region combined with high velocity gradients near the wall (Figure 3 (a) and (b) and Figure 9 (a) and (b)). Further downstream, the axial velocity profile evolves from a flat profile to a less disturbed moderately flat profile at $50.0 D$ (Figure 3 (c) and Figure 9 (c)), and finally to a nearly fully-developed profile at the $105.0 \mathrm{D}$ measurement section (Figure 3 (d) and Figure 9 (d)).

The associated velocity contours of the secondary flow (Figure 3 (e)-(h)) show an overall decrease with increasing distance between the swirl generator and the LDV measurement section. For $\mathrm{Re}=4.0 \cdot 10^{4}$, our results indicate that $\bar{v}_{x y}$ vanishes at around $105.0 \mathrm{D}$ downstream from the swirl disturbance generator (Figure $3(\mathrm{~h})$ and Figure $6(\mathrm{~d})$ ). The maximal magnitudes of the secondary flow are found in the measurement sections at $10.0 \mathrm{D}$ and $12.0 \mathrm{D}$ at the outer part of the core-region (Figure 3 (e) and (f) and Figure $6(a)$ and (b)).

Aside from experiments with $\operatorname{Re}=4.0 \cdot 10^{4}$, we analyze the experiments with $\mathrm{Re}=6.0 \cdot 10^{4}$ at $12.0 \mathrm{D}$ and $50.0 \mathrm{D}$ (Figure $3(\mathrm{i})-(\mathrm{l})$ and Figure 7 (a) and (b)) and $\mathrm{Re}=1.0 \cdot 10^{3}$ at $12.0 \mathrm{D}$ (laminar, Figure $3(\mathrm{~m})$ and $(\mathrm{n})$ ) to inspect the selfsimilarity of flow structures across different flow rates. The turbulent pipe-flow with $\mathrm{Re}=6.0 \cdot 10^{4}$ shows axial and radial velocity profiles similar to the ones of the $\operatorname{Re}=4.0 \cdot 10^{4}$ case. Additionally, we find qualitatively comparable asymmetries for $\operatorname{Re}=4.0 \cdot 10^{4}$ and $\operatorname{Re}=6.0 \cdot 10^{4}$ at the $12.0 \mathrm{D}$ and the $50.0 \mathrm{D}$ measurement sections. It is important to note that the asymmetries that we observe in the flow field include contributions associated with uncertainties in the positioning of the axial pipe center.

The axial velocity profile in the laminar case is less flat and a smaller region in the pipe center appears to attain the peak velocity. The secondary flow reaches lower values than for $\operatorname{Re}=4.0 \cdot 10^{4}$ and $\operatorname{Re}=6.0 \cdot 10^{4}$. This confirms that swirl disturbances decay rapidly for laminar flow conditions.

In panels (a)-(d) of Figure 9, we show a comparison of individual axial velocity profiles at different downstream locations along with the Gersten and Herwig [10] reference profile for a fully-developed turbulent pipe-flow. Analogous data for the magnitude of the secondary flow is shown in panels (a)-(d) of Figure 6. In panel (d) of Figure 9 , the peak velocity at $105.0 D$ takes values around $\bar{w} / w_{\mathrm{vol}} \approx 1.2$, signalizing compliance with an almost fullydeveloped flow profile. The secondary flow profiles show peaks at the outer core region and a linear radial dependency within the core region. This indicates that the swirling flow in the core region has a characteristic motion pattern close to a solid body rotation.

The comparison in Figure 9 (a)-(d) shows that the axial velocity profile relaxes towards the Gersten \& Herwig reference profile with increasing distance from the swirl disturbance generator. The asymmetry persists for the measurements at the $50.0 \mathrm{D}$ and $105.0 \mathrm{D}$ downstream sections. The secondary flow (Figure 6 (a)-(d)) decreases rapidly and nearly vanishes at $105.0 D$, which implies that a fully-developed axial profile is guaranteed after a distance of $105.0 D$ downstream from the swirl disturbance generator.

\subsection{Downstream relaxation of performance indicators}

\subsubsection{Swirl angle}

Following Yeh and Mattingly [39], the level of swirl can be measured quantitatively through (10). Geometrically, the swirl angle (10) is the angle between the ideal velocity vector and the actual velocity vector with swirl. However, the precise definition of the swirl angle may vary slightly depending on the author and may lead to discrepancies in computed values. For example, it is also possible to compute the swirl angle with a tangentially projected velocity $\bar{v}_{t}$ rather than the magnitude of the secondary flow. For a perfectly symmetric flow field, $\bar{v}_{x y}=\bar{v}_{t}$ holds, but this is not necessarily true in practice. Additionally, taking the 
(a) $z / D=10.0$

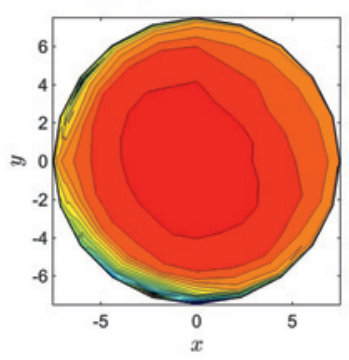

(e) $z / D=10.0$

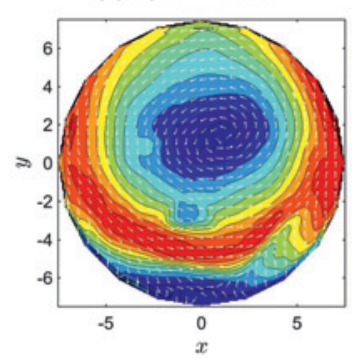

(i) $z / D=12.0$

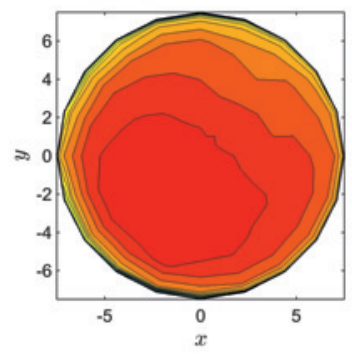

(k) $z / D=12.0$

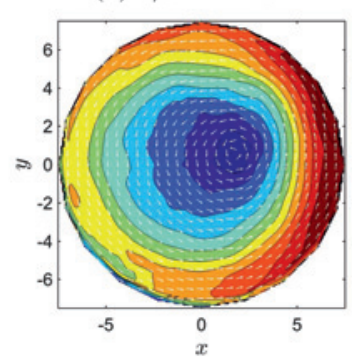

(b) $z / D=12.0$

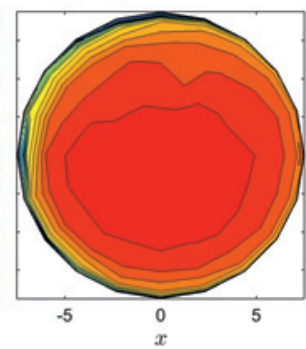

(f) $z / D=12.0$

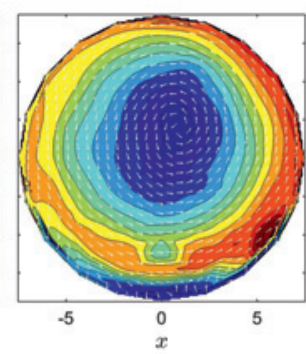

(j) $z / D=50.0$

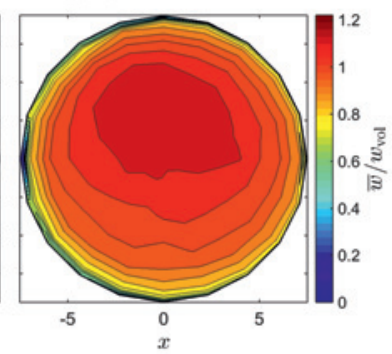

(1) $z / D=50.0$

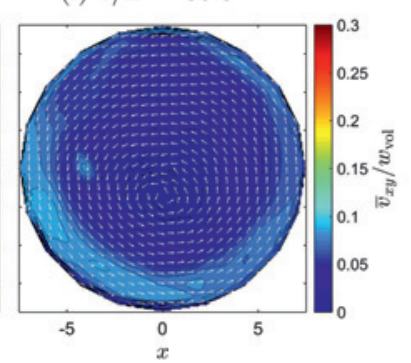

(c) $z / D=50.0$

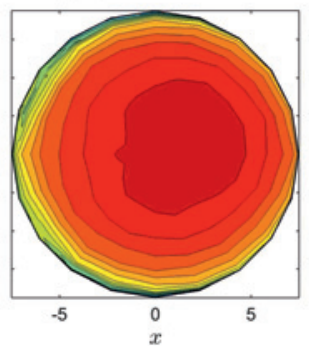

(g) $z / D=50.0$

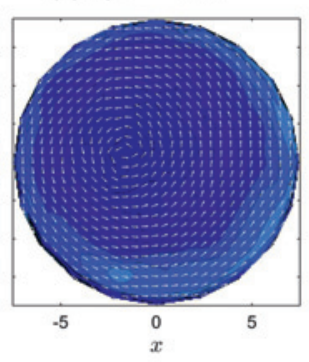

(d) $z / D=105.0$

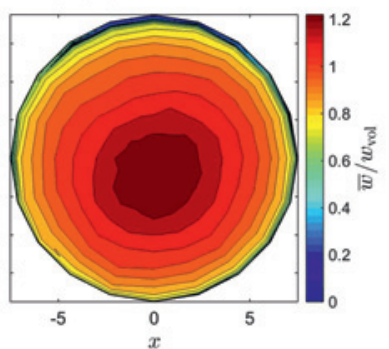

(h) $z / D=105.0$

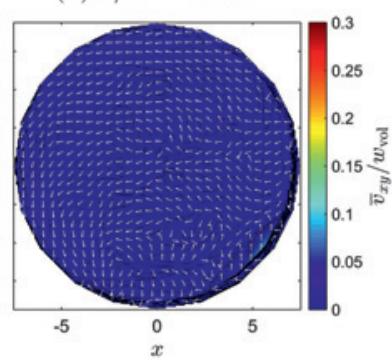

(m) $z / D=12.0$

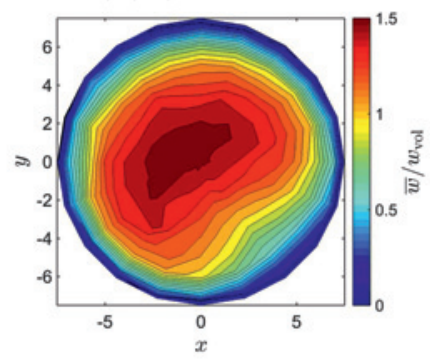

(n) $z / D=12.0$

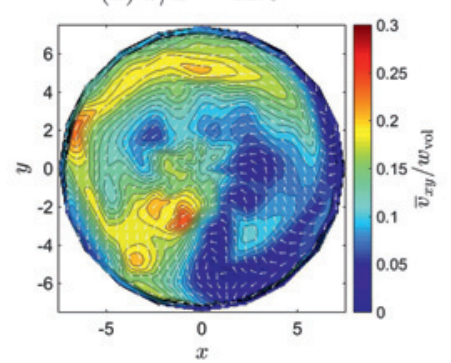

Figure 3: Contour plots of the axial velocity component $\bar{w}$ and the magnitude of the secondary flow $\bar{v}_{x y}$ along with the velocity vectors of the secondary flow for different downstream distances and different Reynolds numbers. The perspective is looking against the primary flow direction (a)-(h) $\operatorname{Re}=4.0 \cdot 10^{4}$, (i)-(l) $\operatorname{Re}=6.0 \cdot 10^{4}$, and (m)-(n) $\operatorname{Re}=1.0 \cdot 10^{3}$.

maximum value of a velocity field may not be very robust and depend on the spatial resolution of experiments or simulation. Hence, the comparability across independent studies is limited.

A comparison of the swirl angle at different downstream locations with the experimental results of Eichler and Lederer [6] and Müller et al. [20] is shown in Figure 4. Eichler and Lederer [6] and Müller etal. [20] use swirl disturbance generators designed according to the same standard.
For downstream locations within the range $0<z / D \leq$ 13.0, the $\operatorname{Re}=6.0 \cdot 10^{4}$ and $\operatorname{Re}=4.0 \cdot 10^{4}$ cases exhibit higher swirl angles than the experimental references, despite the lower Reynolds numbers. At downstream locations close to the swirl disturbance generator, only the laminar case with $\operatorname{Re}=1.0 \cdot 10^{3}$ shows smaller swirl angles than the references. Although locally turbulent zones may be present, this faster decay is consistent with a laminar flow. As discussed in Section 3.2.1, subtleties in the definition of the swirl angles can lead to discrepancies in 


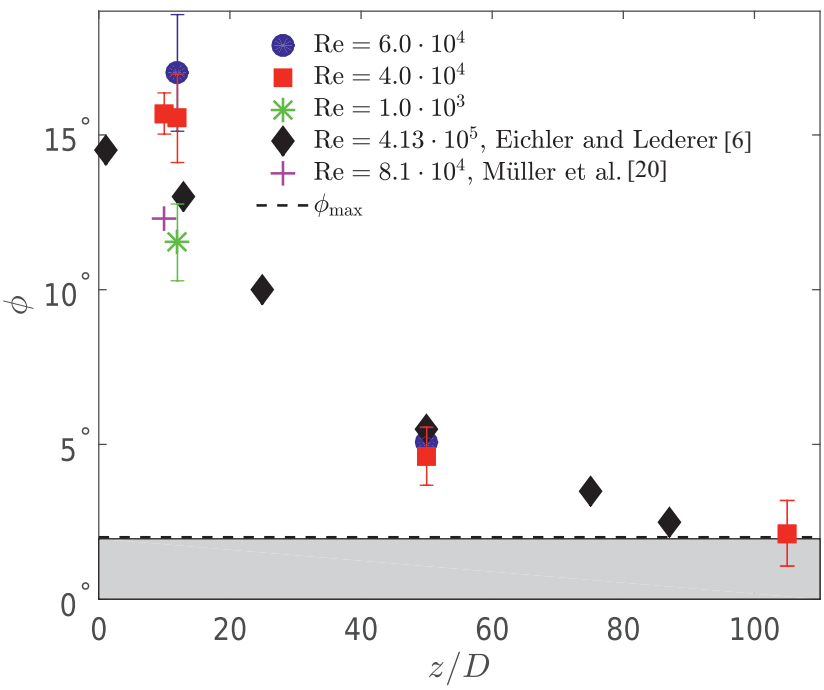

Figure 4: Swirl angles at various downstream distances from the inlet and Reynolds numbers.

computed values. Additionally, manufacturing uncertainties and other parameters like surface roughness are likely to have a significant impact on the experimental boundary conditions and, hence, on the results. The differences between various references confirm that even standardized parts do not necessarily guarantee the reproducibility of experimental boundary conditions.

In contrast, further downstream, the swirl angles of the present experiments with $\operatorname{Re}=6.0 \cdot 10^{4}$ and $\operatorname{Re}=4.0$. $10^{4}$ appear to be in reasonable agreement with the results of Eichler and Lederer [6]. This shows that the Reynolds number and the experimental boundary conditions have a major impact on the swirl angle at locations close to the disturbance generator, but becomes less important further downstream.

A direct comparison of the swirl angle $12.0 \mathrm{D}$ downstream for different Reynolds numbers suggest a linear increase of $\phi$ with increasing Re (Figure 5). The individual profiles (Figure 5 (a)-(c)) show maximal values of around $\bar{v}_{x y, \max } / w_{\mathrm{vol}}=0.2$ for the laminar case, $\bar{v}_{x y, \max } / w_{\mathrm{vol}}=0.3$ for $\operatorname{Re}=4.0 \cdot 10^{4}$, and $\bar{v}_{x y, \max } / w_{\text {vol }}>0.35$ for $\operatorname{Re}=6.0 \cdot 10^{4}$. The standard error of the measurements indicated through the shaded profile is highest for the laminar case, which reflects the difficulty of obtaining a sufficiently good LDV signal at low flow rates with a reasonable amount of flow seeding.

Next, we compare the downstream development of the swirl angle computed from our experiments with the theoretical approximations of Murakami et al. [21], Steenbergen and Voskamp [32], and Gersten and Papenfuss [11]. The objective is to determine whether the theories of $\mathrm{Mu}$ rakami et al. [21], Steenbergen and Voskamp [32], and Gersten and Papenfuss [11] are applicable to the swirl generated with a standardized swirl disturbance generator according to EN ISO 4064-2:2014 [7] and OIML R 49-2:2013 [23]. In particular, we assess whether one measurement at a certain downstream location is sufficient to predict the evolution of the swirl angle using the theories of Murakami et al. [21], Steenbergen and Voskamp [32], and Gersten and Papenfuss [11].

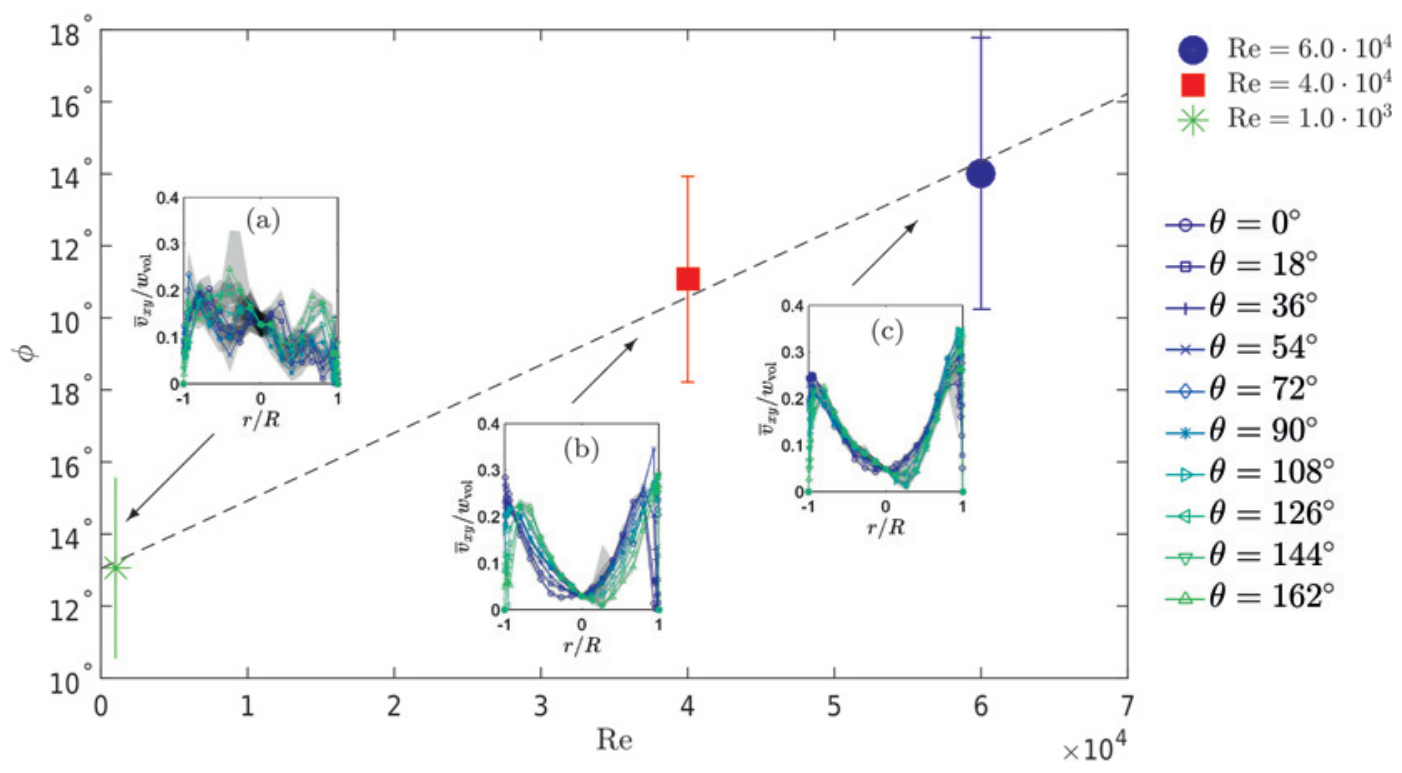

Figure 5: The swirl angle at $12.0 \mathrm{D}$ downstream from the swirl disturbance generator increases linearly with the Reynolds number. The associated profiles of the secondary flow $\bar{v}_{x y} / w_{\text {vol }}$ are shown in insets (a)-(c). 

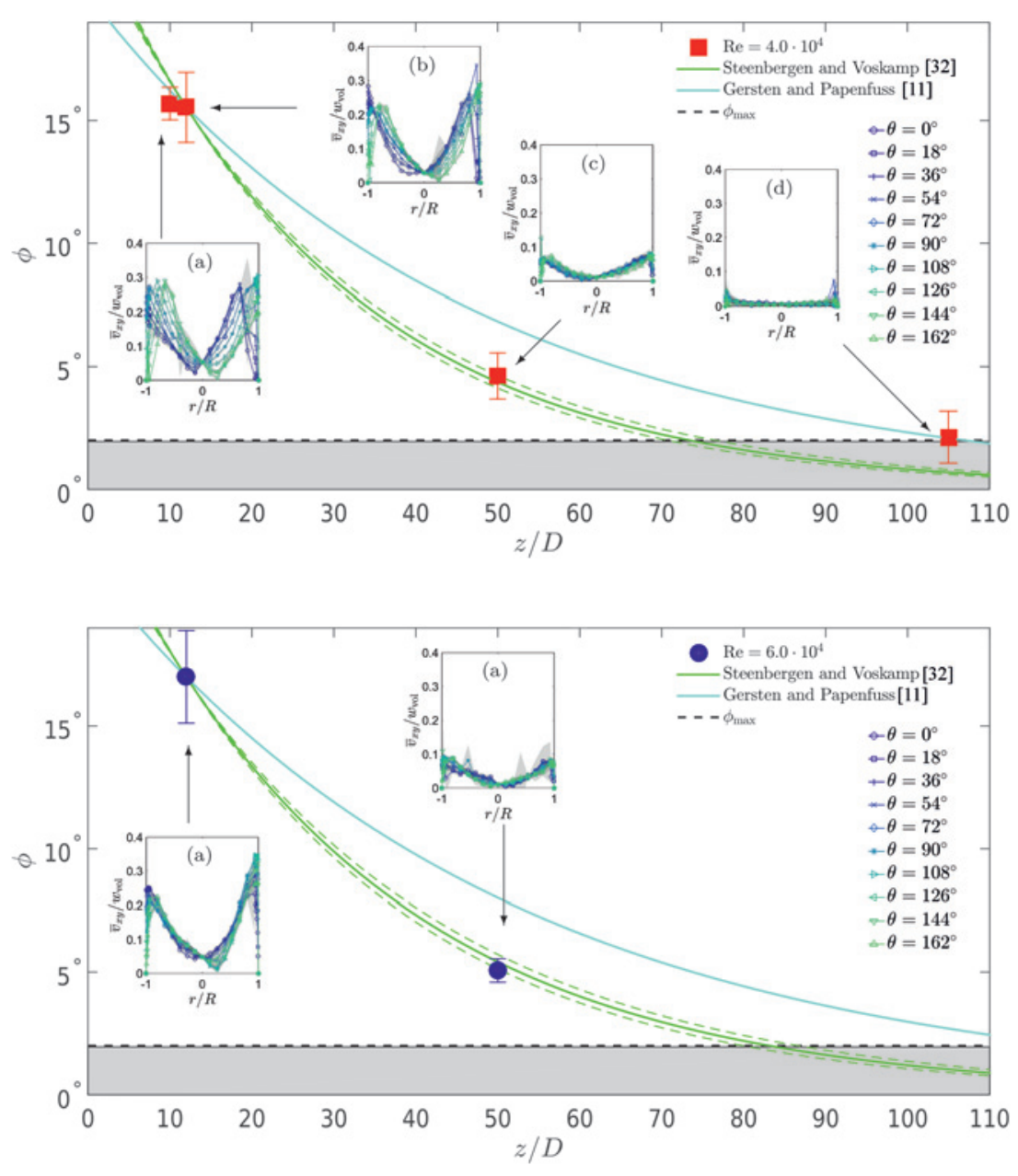

Figure 6: At $\operatorname{Re}=4.0 \cdot 10^{4}$ the present experiments are best approximated by the theory of Steenbergen and Voskamp [32]: Downstream development of $\phi$ and profiles of the secondary flow $\bar{v}_{x y} / w_{\text {vol }}$.
Figure 7: At $\operatorname{Re}=6.0 \cdot 10^{4}$ the present experiments are best approximated by the theory of Steenbergen and Voskamp [32]: Downstream development of $\phi$ and profiles of the secondary flow $\bar{v}_{x y} / w_{\text {vol }}$.
For the comparison, we follow Gersten and Papenfuss [11] and use a slight generalization of (13) so that

$$
S=S_{z_{0}} e^{-\beta\left(z-z_{0}\right) / D},
$$

where $S_{z_{0}}$ is the swirling intensity at the location $z_{0}$. Notice that the generalization (24) is included in the theory of Gersten and Papenfuss [11] to allow for boundary conditions at measurement locations $z_{0} \neq 0$. The decay parameter $\beta$ in (24) is estimated from the theories of Murakami et al. [21], Steenbergen and Voskamp [32], and Gersten and Papenfuss [11]. Steenbergen and Voskamp [32] point out that the swirl angle is a reasonable approximation of the swirl number in the stage of decay where the specific details of the swirl generating process have vanished. Hence, we assume that the swirl angle $\phi$ is a suitable measure for the swirling intensity such that the decay law (24) can be applied to describe the decay of $\phi$ yielding

$$
\phi=\phi_{z_{0}} e^{-\beta\left(z-z_{0}\right) / D} .
$$

For brass pipes and $\operatorname{Re}=2.5 \cdot 10^{5}$ Murakami et al. [21] estimate the parameters in (14) as

$$
2 k \lambda=0.0069
$$

and

$$
V_{\Theta_{0}} V_{0} / S=[1.4,2.0] .
$$

In view of (27) it is reasonable to assume that

$$
V_{\Theta_{0}} V_{0} / S \approx 1.7 \pm 0.3
$$

and, consequently,

$$
\beta_{\mathrm{M}}=0.0117 \pm 0.0021,
$$

where the index M in (29) is used to refer to Murakami et al. [21]. Notice that the theory of Murakami et al. [21] is only applicable for $\operatorname{Re}=2.5 \cdot 10^{5}$.

Steenbergen and Voskamp [32] suggest that the coefficient $\beta$ in turbulent swirling flows in smooth pipes can be 
Table 2: Swirl number $S^{*}$ according to (2) at various distances downstream from the swirl disturbance generator for different Reynolds numbers.

\begin{tabular}{lrrrr}
\hline & $\mathbf{1 0 . 0 D}$ & $\mathbf{1 2 . 0 D}$ & $\mathbf{5 0 . 0 D}$ & $\mathbf{1 0 5 . 0 D}$ \\
\hline $\operatorname{Re}=6.0 \cdot 10^{4}$ & - & 0.1244 & 0.0361 & - \\
$\operatorname{Re}=4.0 \cdot 10^{4}$ & 0.0978 & 0.0945 & 0.0300 & 0.0079 \\
$\operatorname{Re}=1.0 \cdot 10^{3}$ & - & 0.0499 & - & - \\
\hline
\end{tabular}

computed by

$$
\beta_{\mathrm{S}}=(1.49 \pm 0.07) \lambda
$$

for

$$
0 \leq S^{*} \leq 0.18,
$$

where $\lambda$ is the dimensionless friction coefficient for fully developed pipe flow and the index $S$ is used to refer to Steenbergen and Voskamp [32]. To check if the criterion (31) holds, we compute (2) for the present experiments. We find that the criterion (31) applies to all experiments, as summarized in Table 2.

Since the threshold (31) is not exceeded, (30) can be used to compute $\beta$. The dimensionless friction coefficient for fully developed pipe flow is

$$
\lambda=\frac{8 \tau_{\text {wall }}}{\rho w_{\text {vol }}^{2}}
$$

with $\tau_{\text {wall }}$ the wall shear stress and $\rho$ the density of the fluid. For a smooth pipe, $\tau_{\text {wall }}$ can be computed from the empirical Blasius equation (Blasius [4])

$$
\frac{2 \tau_{\text {wall }}}{\rho w_{\text {vol }}}=\frac{0.0791}{\mathrm{Re}^{0.25}}
$$

for turbulent flow and from

$$
\frac{2 \tau_{\text {wall }}}{\rho w_{\text {vol }}}=\frac{16}{\operatorname{Re}}
$$

for laminar flow. In view of (30), (32), and (33), we find

$$
\beta_{\mathrm{S}}=0.0334 \pm 0.0015 \text { for } \operatorname{Re}=4.0 \cdot 10^{4} .
$$

Values for $\beta_{\mathrm{S}}$ at different Reynolds numbers are computed analogously and summarized in Table 3 for all experiments.

The theory of Gersten and Papenfuss [11] is based on solving the incompressible NS equations with an asymptotic expansion approach and yields an exponential decay of swirl disturbances in turbulent pipe-flow. Adopting the theory of Gersten and Papenfuss [11] for the special case of a single longitudinal vortex, the rate of decay is proportional to the dimensionless friction factor $\lambda$ such that

$$
\beta_{\mathrm{P}}=\lambda
$$

where the index $P$ is used to refer to Gersten and Papenfuss [11] and $\lambda$ is determined from the empirical relationship (Zagarola and Smits [41])

$$
\frac{1}{\sqrt{\lambda}}=1.869 \log (\operatorname{Re} \sqrt{\lambda})-0.241
$$

for high Reynolds numbers. Solving (37) numerically for $\operatorname{Re}=4.0 \cdot 10^{4}$ yields $\lambda=0.0216$ and, hence,

$$
\beta_{\mathrm{P}}=0.0216 \text { for } \mathrm{Re}=4.0 \cdot 10^{4} .
$$

Values for $\beta_{\mathrm{P}}$ at different Reynolds numbers are computed analogously and summarized in Table 3 for all experiments.

A comparison of the $\mathrm{Re}=4.0 \cdot 10^{4}$ case with the theories of Steenbergen and Voskamp [32] and Gersten and Papenfuss [11] is shown in Figure 6. Here, we choose the boundary condition at the location $z_{0}=12.0 \mathrm{D}$ with $\phi_{z_{0}}=15.54^{\circ}$. The theory of Gersten and Papenfuss [11] predicts a slower decay than the theory of Steenbergen and

Table 3: Dimensionless swirl decay coefficients from the theories of Murakami et al. [21], Steenbergen and Voskamp [32], and Gersten and Papenfuss [11] for the Reynolds numbers of the present experiments $\left(\operatorname{Re}=6.0 \cdot 10^{4}, \operatorname{Re}=4.0 \cdot 10^{4}\right.$, and $\left.\operatorname{Re}=1.0 \cdot 10^{3}\right)$ and the Reynolds numbers of the references used for comparison.

\begin{tabular}{lrrr}
\hline & $\begin{array}{r}\text { Murakami et al. [21] } \\
\boldsymbol{\beta}_{\mathrm{M}}\end{array}$ & $\begin{array}{r}\text { Steenbergen and Voskamp [32] } \\
\boldsymbol{\beta}_{\mathrm{S}}\end{array}$ & $\begin{array}{r}\text { Gersten and Papenfuss [11] } \\
\boldsymbol{\beta}_{\mathrm{P}}\end{array}$ \\
\hline $\operatorname{Re}=6.0 \cdot 10^{4}$ & - & $0.0301 \pm 0.0014$ & 0.0198 \\
$\operatorname{Re}=4.0 \cdot 10^{4}$ & - & $0.0334 \pm 0.0015$ & 0.0216 \\
$\operatorname{Re}=1.0 \cdot 10^{3}$ & - & $0.0784 \pm 0.0037$ & 0.0567 \\
$\operatorname{Re}=4.13 \cdot 10^{5}$ (Eichler and Lederer [6]) & - & $0.0186 \pm 0.0009$ & 0.0138 \\
$\operatorname{Re}=8.1 \cdot 10^{4}$ (Müller et al. [20]) & - & $0.0280 \pm 0.0013$ & 0.0187 \\
$\operatorname{Re}=2.5 \cdot 10^{5}$ (Murakami et al. [21]) & $0.0117 \pm 0.0021$ & $0.0211 \pm 0.0010$ & 0.0151 \\
\hline
\end{tabular}




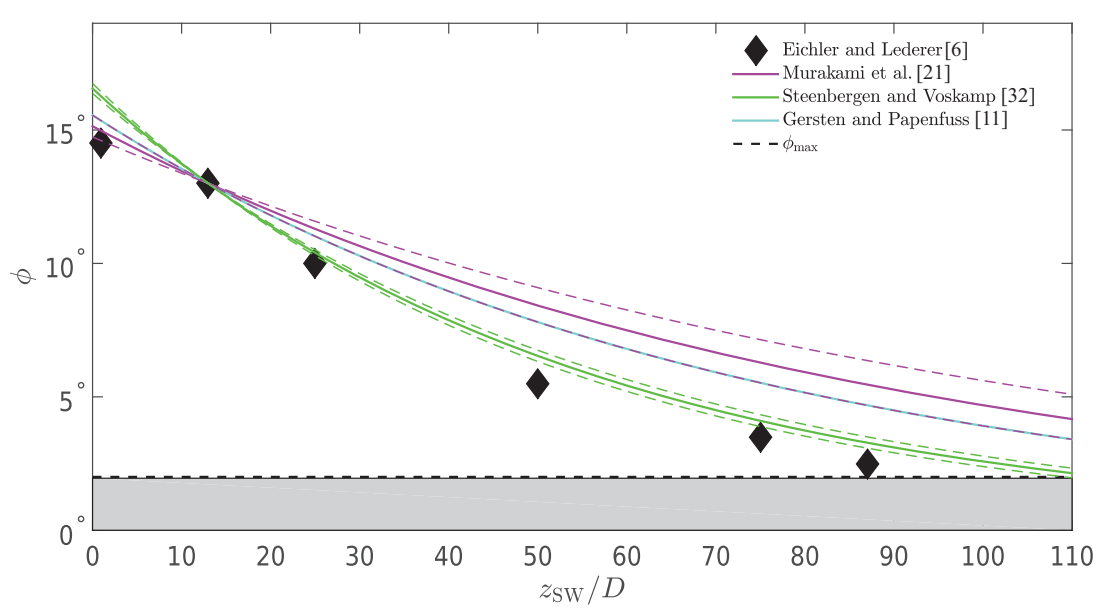

Figure 8: Comparison of Steenbergen and Voskamp [32] and Gersten and Papenfuss [11] for $\mathrm{Re}=4.13 \cdot 10^{5}$ and Murakami et al. [21] for $\mathrm{Re}=2.5 \cdot 10^{5}$ with the experimental results of Eichler and Lederer [6].
Voskamp [32]. While the theory of Gersten and Papenfuss [11] provide a good prediction of the experimental results at $10.0 \mathrm{D}$ and $105.0 \mathrm{D}$, the value at $50.0 \mathrm{D}$ is not captured accurately. In contrast, the theory of Steenbergen and Voskamp [32] provides good agreement at intermediate downstream locations, whereas it predicts high values at $10.0 \mathrm{D}$ and low values at $105.0 \mathrm{D}$. However, inspection of the individual profiles in Figure 6 (d) shows that there are only a few peaks in the $\theta=0^{\circ}$ profile whereas the other profiles of the secondary flow are practically flat. This is also reflected in the larger error bar of the swirl angle at 105.0D which illustrates the importance of full two-dimensional measurements instead of individual paths, since this information would be inaccessible otherwise.

An analogous comparison of the $\mathrm{Re}=6.0 \cdot 10^{4}$ case with the theories of Steenbergen and Voskamp [32] and Gersten and Papenfuss [11] is shown in Figure 7. Here, we choose the boundary condition at the location $z_{0}=$ $12.0 D$ with $\phi_{z_{0}}=17.0^{\circ}$. The two theories show the same qualitative behavior as in Figure 6. The theory of Steenbergen and Voskamp [32] accurately predicts the experimental results at $50.0 D$ downstream.

An analogous comparison of the experimental results from Eichler and Lederer [6] $\left(\mathrm{Re}=4.13 \cdot 10^{5}\right)$ with the theories of Steenbergen and Voskamp [32] and Gersten and Papenfuss [11] is shown in Figure 8. Additionally, the theory of Murakami et al. [21] with a slightly different Reynolds number is included. The theory of Murakami et al. [21] predicts the slowest decay although the lower bound with the estimate $V_{\Theta_{0}} V_{0} / S=2.0$ is close to the theory of Gersten and Papenfuss [11]. In consistency with the present experiments, the theory of Steenbergen and Voskamp [32] also provides the best prediction of the experiments by Eichler and Lederer [6]. Consequently, the theory of Steenbergen and Voskamp [32] yields the best overall predictions of the swirl angle generated with a standardized swirl disturbance generator. Next, we compare the performance indicators of the axial velocity profile.

\subsubsection{Profile factor}

Following Yeh and Mattingly [38], the dimensionless profile factor $K_{p}$ is defined as

$$
K_{p}=\frac{K_{p, m}}{K_{p, s}},
$$

with

$$
K_{p, m}=\frac{1}{w_{\mathrm{vol}} D} \int_{-R}^{R}\left(w_{m}-\bar{w}\right) \mathrm{d} r
$$

and

$$
K_{p, s}=\frac{1}{w_{\mathrm{vol}} D} \int_{-R}^{R}\left(w_{m, s}-w_{s}\right) \mathrm{d} r,
$$

where $w_{m}=\bar{w}(r=0)$ is the velocity at the pipe center, $w_{m, s}$ is the velocity of the norm profile at the pipe center, and $w_{s}$ is the norm velocity profile. The profile factor is a measure for peakness $\left(K_{p}>1\right)$ or flatness $\left(K_{p}<1\right)$ of measured velocity profiles with respect to standard profiles such as Hagen-Pouiseuille for laminar flow or Gersten and Herwig [10] for turbulent flow. Notice that the Equations (39), (40), and (41) are defined for individual paths and averaged over all paths on the measurement grid, as described in Section 2.2.

Figure 9 shows the profile factor $K_{p}$ (panel (e)) and the asymmetry factor $K_{a}$ (panel (f)) along with the individual axial velocity profiles (panels (a)-(d)). The admissible ranges for an approximately fully developed flow profile 
(a) $z / D=10.0$

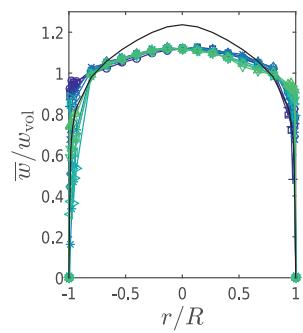

(b) $z / D=12.0$

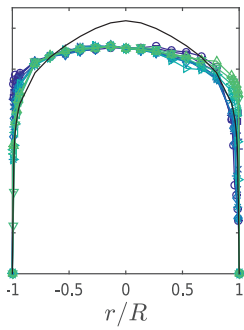

(e)

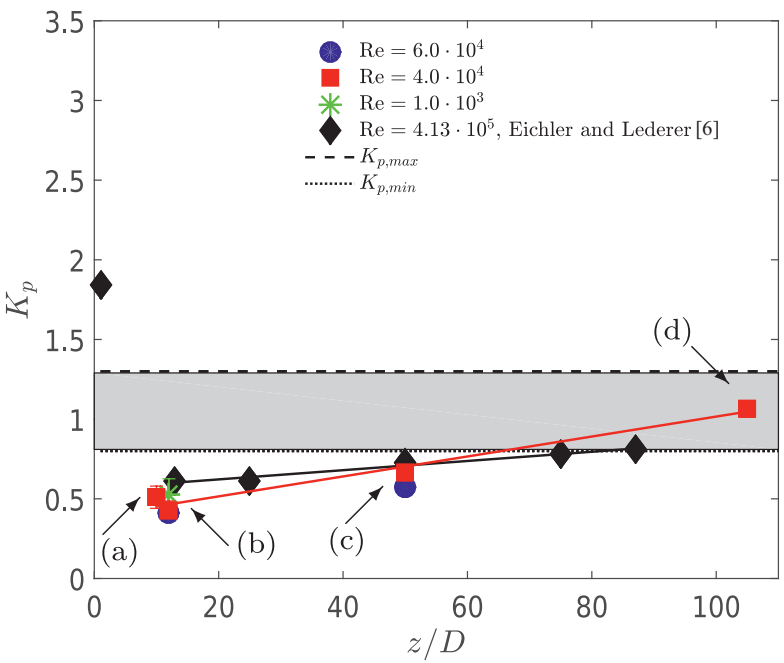

(d) $z / D=105.0$

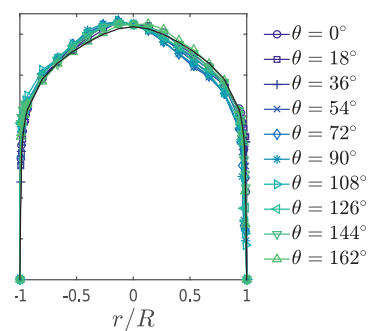

(f)

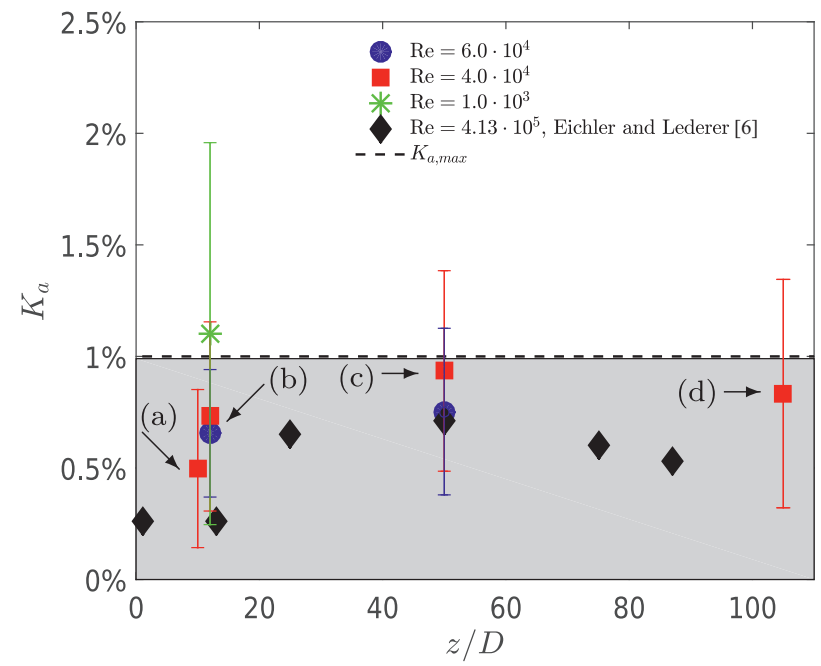

Figure 9: Downstream development of the profile factor $K_{p}$ and the asymmetry factor $K_{a}$ for different Reynolds numbers ((e) and (f)) and profiles of the axial flow for $\operatorname{Re}=4.0 \cdot 10^{4}((\mathrm{a})-(\mathrm{d}))$.

are bounded by $K_{p, \max }=1.3, K_{p, \min }=0.8$, and $K_{a, \max }=$ $1.0 \%$, as indicated through the shaded areas in Figure 9. For small downstream distances, the profile factor of all experimental results is below the threshold $K_{p \text {,min }}$, which confirms that the swirl disturbance generator provides notable disturbances of axial velocity profiles in addition to the secondary flow. Further, these disturbances persist for long downstream distances. The profile factor for $\mathrm{Re}=$ $6.0 \cdot 10^{4}$ appears to relax slower in comparison to the experiments with $\operatorname{Re}=4.0 \cdot 10^{4}$ and the experiments of Eichler and Lederer [6]. For $\operatorname{Re}=4.0 \cdot 10^{4}$, our results show an approximately linear dependency of $K_{p}$ with increasing $z / D$, suggesting that

$$
K_{p} \approx \zeta z / D+K_{p_{0}}
$$

where $\zeta$ is the slope and $K_{p_{0}}$ is the boundary condition for the profile factor at $z / D=0$. A fit of our results yields

$$
\begin{array}{ll}
\zeta & =0.00626 \pm 0.00317 \\
K_{p_{0}} & =0.39010 \pm 0.18570
\end{array}
$$

for $\mathrm{Re}=4.0 \cdot 10^{4}$. Similarly, a fit of the experimental results of Eichler and Lederer [6] for $z / D>2.0$ yields

$$
\begin{array}{ll}
\zeta & =0.00289 \pm 0.00104 \\
K_{p_{0}} & =0.56400 \pm 0.05990
\end{array}
$$

Notice that the experiments of Eichler and Lederer [6] indicate a very high $K_{p}$ at $z / D=2.0$, suggesting that the linear dependency is only a reasonable approximation for intermediate and long downstream distances. Short downstream locations were inaccessible in the present experimental setup. Importantly, the profile factor relaxes only linearly towards acceptable values within the established bounds, whereas the swirl angle relaxes exponentially. 
(e)

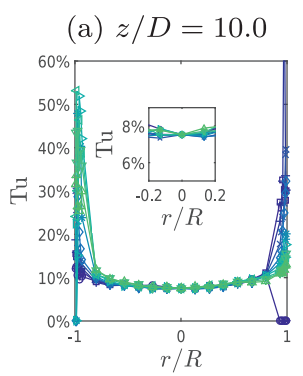

(b) $z / D=12.0$

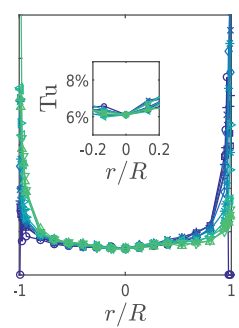

(c) $z / D=50.0$

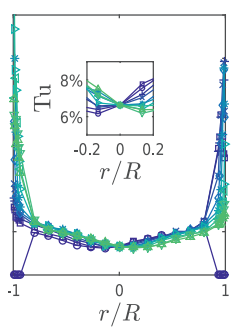

(d) $z / D=105.0$

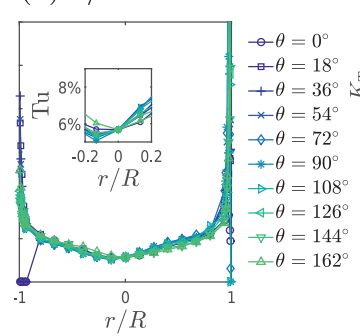

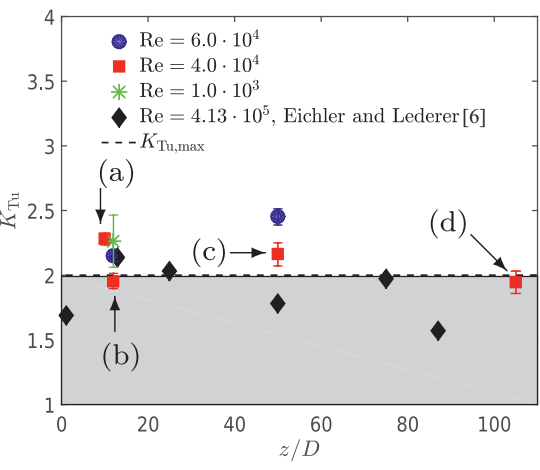

Figure 10: Downstream development of the turbulence factor $K_{\mathrm{Tu}}$ for different Reynolds numbers (e) and profiles of the turbulence intensity ((a)-(d)) at corresponding distances for $\mathrm{Re}=4.0 \cdot 10^{4}$.

\subsubsection{Asymmetry factor}

Following Yeh and Mattingly [38], the asymmetry factor

$$
K_{a}=\frac{1}{D} \frac{\int_{-R}^{R} r \bar{w} \mathrm{~d} r}{\int_{-R}^{R} \bar{w} \mathrm{~d} r}
$$

quantifies the relative radial displacement of the center of gravity of the area under the flow profile with respect to the pipe center.

For the asymmetry factor we find the highest value for the measurement in the cross-section located at 50.0D. Only the laminar measurement shows a $K_{a}$ higher than $1.0 \%$, pointing towards the presence of a significant asymmetry in the axial flow profile. There is no clear dependency indicating a relaxation of the asymmetry factor.

\subsubsection{Turbulence factor}

Each LDV point measurement is a collection of a large number of bursts resulting in a histogram (or probability density function) for the velocity. The level of dispersion (i.e. the standard deviation) of this histogram quantifies the turbulence intensity (18). As discussed by Durst et al. [5] and generalized by Pashtrapanska [25], the turbulence intensity (18) in the core region $-0.2 \leq r / R \leq 0.2$ can be estimated as

$$
\mathrm{Tu}_{s}=0.13\left(\operatorname{Re} \frac{w_{m, s}}{w_{\mathrm{vol}}}\right)^{-1 / 8}
$$

for $\operatorname{Re} \frac{w_{m, s}}{w_{\mathrm{vol}}} \geq 4500$. The turbulence factor $K_{\mathrm{Tu}}$ is defined as

$$
K_{\mathrm{Tu}}=\frac{\mathrm{Tu}_{\mathrm{max}}}{\mathrm{Tu}_{\mathrm{s}}},
$$

where $\mathrm{Tu}_{\max }$ is the maximum of (18) in the core region $-0.2 \leq r / R \leq 0.2$.

The turbulence factor $K_{\mathrm{Tu}}$ is shown in Figure 10 (e) along with the turbulence profiles (panels (a)-(d)). The experimental results of Eichler and Lederer [6] indicate a decrease in the region from $12.0 D$ to $50.0 D$ whereas the present experiments indicate an increase for the same region. The experiments of Eichler and Lederer [6] reach an acceptable turbulence factor at $50.0 \mathrm{D}$ but the present experiments beyond 105.0D. Furthermore, Eichler and Lederer [6] find a relatively low $K_{\mathrm{Tu}}$ at $87.0 \mathrm{D}$ whereas the present experiments at $\operatorname{Re}=4.0 \cdot 10^{4}$ show a high $K_{\mathrm{Tu}}$ at $105.0 D$. Consequently, $K_{\mathrm{Tu}}$ appears to comply with the established guidelines at distant downstream locations, but the relaxation does not show a clear tendency. However, for a realistic interpretation of results, it is important to realize that the accuracy of $K_{\mathrm{Tu}}$ as a statistical quantity is much more affected by the measurement uncertainty than the velocity measurements themselves.

\section{Conclusions and outlook}

A comparison between various experiments, references, and theories of disturbed pipe-flow is realized using a standardized swirl disturbance generator. We present results for distances $10.0 D, 12.0 D, 50.0 D$, and $105.0 D$ downstream from the swirl disturber, which exhibit higher swirl angles $\phi$ than the experimental results of Eichler and Lederer [6] for distances $z / D \leq 12.0$, but show reasonable agreement further downstream. The theory of Steenbergen 
and Voskamp [32] provides the best overall predictions of the swirl angle. The profile factor $K_{p}$ is found to behave similarly as in the experiments of Eichler and Lederer [6]. We find that $K_{p}$ relaxes linearly toward the fully developed state and reaches the desired range $\left(0.8<K_{p}<1.3\right)$ at distances $z / D>80.0$. The admissible limits of the asymmetry factor $K_{a}$ is only exceeded for laminar flow conditions. In addition, the turbulence factor $K_{\mathrm{Tu}}$ shows a scatter around the maximal value $K_{\mathrm{Tu}}=2.0$ and no systematic relaxation is found.

Due to the good agreement of the theory of Gersten and Papenfuss [11] and Steenbergen and Voskamp [32] for the prediction of swirl angles, our results provide an experimental reference to estimate flow patterns of a standardized swirl disturbance generator.

Acknowledgement: DFH acknowledges the support of Mario Turiso in preparing the manuscript. DFH and SG would like to thank Dr. Jens Lykke Sørensen for providing valuable feedback on earlier versions of the manuscript and Preben Mønsted Nielsen, Salim Umar, Karsten Lundsgaard and Thor Marum for their great technical support.

Funding: Kampstrup A/S

\section{References}

1. Algifri AH (1987) Prediction of the decay process in turbulent swirl flow. Proc Inst Mech Eng 201:279-283

2. Algifri AH, Bhardwaj RK, Rao Y (1988) Heat transfer in turbulent decaying swirl flow in circular pipe. Int J Heat Mass Transfer 31:1563-1568

3. Binnie AM (1957) Experiments on the slow swirling flow of viscous liquid through a tube. Quart J Mech Appl Math 10:276290

4. Blasius H (1913) Das Ähnlichkeitsgesetz bei Reibungsvorgängen in Flüssigkeiten. Forschung Arb Ing-Wes 131

5. Durst F, Fischer M, Jovanović J, Kikura H (1998) Methods to set up and investigate low Reynolds number, fully developed turbulent plane channel flows. J Fluids Eng 120:496-503

6. Eichler T, Lederer T (2015) Flow development behind a swirl generator in a hot-water standard measuring facility for large volume rates. Flow Meas Instr 42:90-97

7. EN ISO 4064-2:2014 (2014) Water meters for cold potable water and hot water - part 2: Test methods

8. EN ISO 5167-1:2003 (2003) Measurement of fluid flow by means of pressure differential devices inserted in circular cross-section conduits running full-Part 1: General principles and requirements

9. Genc BZ, Ertunç Ö, Jovanović J, Delgado A (2009) LDA Messungen von Rohrströhmungen mit Drall für einen breiten Bereich von Drallzahlen. In: Fachtagung Lasermethoden in der Strömungsmechanik, Erlangen, Germany, 8-10 September, 2009
10. Gersten K, Herwig H (1992) Strömungsmechanik: Grundlagen der Impuls-, Wärme- und Stoffübertragung aus asymptotischer Sicht, aufl. 1992 edn. Grundlagen und Fortschritte der Ingenieurwissenschaften, Vieweg \& Teubner, Wiesbaden

11. Gersten K, Papenfuss HD (2005) Decay of disturbances in turbulent pipe flow. Fluid Mechanics of Flow Metering pp 23-46

12. Hinz, DF (2015) Reconstruction of turbulent pipe-flow profiles from laser-Doppler velocimetry data, in: 15th European Turbulence Conference, 25-28 August 2015, Delft, The Netherlands

13. Ito S, Ogawa K, Kuroda C (1979) Decay process of swirling flow in a circular pipe. J Chem Eng Japan 13:6-5

14. Khalatov AA (1977) Integral method for calculating swirled flow development in a channel. AVTEK 20:106-113

15. Kitoh 0 (1991) Experimental study of turbulent swirling flow in a straight pipe. J Fluid Mech 225:445-478

16. Koch R (1958) Druckverlust und Wärmeübertragung bei verwirbelter Strömung. VDI-Forschungsheft B24:469

17. Kreith F, Margolis D (1959) Heat transfer and friction in turbulent vortex flow. Appl Sci Res A8:457-468

18. Kreith F, Sonju OK (1965) The decay of a turbulent swirl in a pipe. J Fluid Mech 22:257-271

19. Migay VK, Golubev LK (1970) Friction and heat transfer in turbulent swirl flow with a variable swirl generator in a pipe. Heat Transf Sov Res 2:68-73

20. Müller U, Dues M, Baumann H (2007) Vollflächige Erfassung von ungestörten und gestörten Geschwindigkeitsverteilungen in Rohrleitungen mittels der Laser-Doppler-Velocimetrie (completely detection of disturbed and undisturbed flow conditions in pipes with laser doppler velocimetry). Tech Mess 74:342352

21. Murakami M, Osami K, Katayama Y, lida Y (1976) An experimental study of swirling flow in pipes. B JSME 19:118-126

22. Nutall JB (1953) Axial flow in a vortex. Nature 172:582-583

23. OIML R 49-2:2013 (2013) International recommendation: Water meters for cold potable water and hot water, part 2: Test methods

24. Parchen RR (1993) Decay of swirl in turbulent pipe flows. Proefschrift TU Eindhoven

25. Pashtrapanska M (2004) Experimentelle Untersuchung der turbulenten Rohrströmung mit abklingender Drallkomponente. $\mathrm{PhD}$ thesis, Friedrich-Alexander-Universität Erlangen-Nürnberg

26. Reader-Harris MJ (1994) The decay of swirl in a pipe. Int J Heat Fluid 15:212-219

27. Rocklage-Marliani G, Schmidts M, Vasanta Ram VI (2003) Three-dimensional laser-doppler velocimeter measurements in swirling turbulent pipe flow. Flow Turb Comb 70:43-67

28. Sachdeva RC (1968) An experimental investigation of swirling flow through a pipe. Master's thesis, Indian Institue of Technology Kampur

29. Senoo Y, Nagata T (1972) Swirl flow in long pipes with different roughness. B JSME 15:1514-1521

30. Smithberg G, Landis F (1964) Friction and forced convection heat transfer characteristics tubes with twisted tape swirl generators. J Heat Transfer 86:39-49

31. Steenbergen W, Parchen RR (1997) An experimental and numerical study of turbulent swirling pipe flows. Trans ASME 120:54-61 
32. Steenbergen W, Voskamp J (1998) The rate of decay of swirl in turbulent pipe flow. Flow Meas Instr 9:67-78

33. Talbot L (1954) Laminar swirling pipe flow. J Appl Mech 10:119126

34. Verein Deutscher Ingeniere (1999) Netzmessungen in Strömungsquerschnitten - Allgemeine Richtlinien und mathematische Grundlagen, VDI/VDE 2640 Blatt 1 (1999)

35. Verein Deutscher Ingeniere (1999) Netzmessungen in Strömungsquerschnitten - Allgemeine Richtlinien und mathematische Grundlagen, VDI/VDE 2640 Blatt 2 (1999)

36. Wolf Jr L, Lavan Z, Fejer A (1969) Measurements of the decay of swirl in turbulent flow. AIAA J 77:971-972

37. Yajnik KS, Subbaiah MV (1973) Experiments on swirling turbulent flow: Part 1 similarity in swirling flows. J Fluid Mech 60:665-687

38. Yeh TT, Mattingly GE (1994) Pipe flow downstream of a reducer and its effects on flowmeters. Flow Meas Instr 5:181-187

39. Yeh TT, Mattingly GE (1995) Laser Doppler velocimeter studies of the pipeflow produced by a generic header. NIST technical note 1409

40. Yu S, Kitoh O (1994) A general formulation for the decay of swirling motion along a straight pipe. Int Commun Heat Mass Trans 21:719-728

41. Zagarola MV, Smits AJ (1998) Mean-flow scaling of turbulent pipe flow. J Fluid Mech 373:33-79

\section{Bionotes}

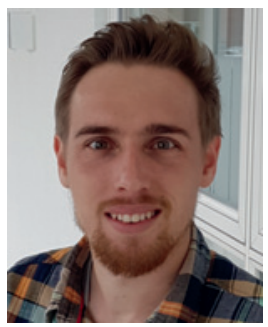

Simon Graner, M.Sc.

Kamstrup A/S,

Industrivej 28, 8660 Skanderborg,

Denmark,

Tel.: +4589931676

sgr@kamstrup.com

Mr. Graner is responsible for optimizing verification processes and implementing novel statistical process control in Kamstrup's flow laboratory in Denmark. Mr. Graner studied mechanical engineering at Technische Universität München and at Pontificia Universidad Católica de Chile in Santiago de Chile. Prior to his current position Mr. Graner wrote his M.Sc. thesis on laser-Doppler velocimetry at Kamstrup in collaboration with the Chair of Aerodynamics and Fluidmechanics at Technische Universität München.

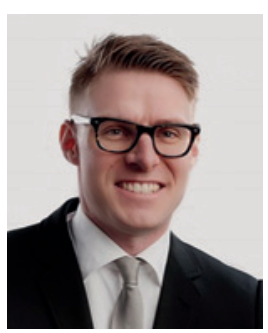

\section{Dr. Denis F. Hinz}

Kamstrup A/S, Industrivej 28, 8660 Skanderborg, Denmark, Tel.: +4589931613 dfh@kamstrup.com

Dr. Hinz is leading a team of engineers responsible for the flow laboratory at Kamstrup's headquarters in Denmark. His main research interests are experimental and computational fluid dynamics with focus on uncertainty quantification, optimization, and metrology. Prior to joining Kamstrup, Dr. Hinz worked at the Okinawa Institute of Science and Technology in Japan. He holds a Ph.D. in mechanical engineering from McGill University in Canada.

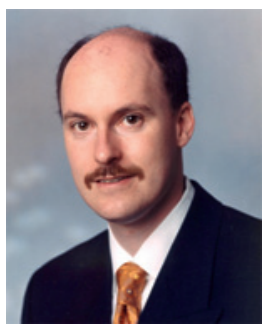

apl. Prof. Dr.-Ing. Christian Breitsamter Technical University of Munich, Chair of Aerodynamics and Fluidmechanics, Boltzmannstrasse 15, 85748 Garching, Germany Christian.Breitsamter@aer.mw.tum.de

Prof. Breitsamter is head of the Aircraft and Spacecraft Aerodynamics Division at the Chair of Aerodynamics and Fluidmechanics. Research projects are on wind tunnel testing of aircraft and rotorcraft configurations with a special emphasis on flow control including a variety of optical measurement techniques. A further focus is on the development of aeroelastic simulation tools and analysis of complex vortex dominated flows. 